\title{
A Sliding Mode Regulator for Antilock Brake System *
}

\author{
Juan Diego Sánchez-Torres*, Alexander G. Loukianov*, \\ Marcos I. Galicia* and Jorge Rivera ${ }^{* *}$ \\ * Department of Electrical Engineering and Computer Science, \\ Automatic Control Laboratory, CINVESTAV Unidad Guadalajara, \\ Zapopan, Jalisco, 45015 México. \\ (e-mail: [dsanchez, louk, mgalicia]@gdl.cinvestav.mx) \\ ** Centro Universitario de Ciencias Exactas e Ingenierías de la \\ Universidad de Guadalajara, Guadalajara, Jalisco, 44430 México. \\ (e-mail: jorge.rivera@cucei.udg.mx)
}

\begin{abstract}
A Sliding Mode (SM) Regulator is proposed for an Antilock Brake System (ABS) control problem by employing Block Control SM and regulation concepts. This regulator has robustness against matched and unmatched perturbations, and the capability to estimate and incorporate the residual dynamics. Numeric simulations are carried on in order to verify the closed-loop system performance, where simulations predict the robustness of the ABS to matched and unmatched perturbations.
\end{abstract}

Keywords: Sliding Mode Control, Brake Control, Antilock Braking Systems (ABS)

\section{INTRODUCTION}

The ABS control problem consists of imposing a desired vehicle motion and as a consequence, provides adequate vehicle stability. The main difficulty arising in the ABS design is due to its high nonlinearities and uncertainties presented in the mathematical model. Therefore, the ABS has become an attractive research area in nonlinear systems control framework. There are several works reported in the literature using the sliding mode technique, some examples are Tan and Chin [1991], Drakunov et al. [1995], Unsal and Pushkin [1999], Hadri et al. [2002], MingChin and Ming-Chang [2003].

Despite of the mentioned above works we consider a real situation: the control input can take only two values "0" or "1" that corresponds to the control valve position.

In this work, we are compelled with asymptotically tracking the relative slip to a desired trajectory. To solve this problem we propose to use the block control technique combined with the SM control algorithm to achieve robustness to perturbations, and ensure asymptotically output tracking along with the stabilization of the residual dynamic consisting of the vehicle velocity. In order to accomplish such tasks, we take the following steps:

- first, the block control is used to linearize and asymptotically stabilize the output error dynamics, and derive a standard sliding variable,

- secondly, it is defined the velocity error as a difference between real vehicle velocity and an auxiliary integral variable which estimates the vehicle velocity steady state value on the central manifold; finally,

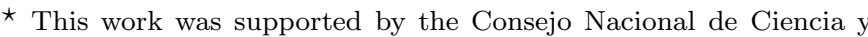
Tecnología de México (CONACYT) under grant 46069.
- following this procedure a sliding manifold is formed as a linear combination of the standard sliding variable and the velocity error and the SM control is implemented to ensure this manifold to be attractive.

As a result, the vehicle dynamic, i.e., the vehicle velocity, on the designed SM manifold becomes asymptotically stable, ensuring an stable tracking error.

\section{MATHEMATICAL MODEL}

In this section, the dynamic model of a vehicle is shown. Here we use a quarter of vehicle model, this model considers the pneumatic brake system, the wheel motion and the vehicle motion. We study the task of controlling the wheels rotation, such that, the longitudinal force due to the contact of the wheel with the road, is near from the maximum value in the period of time valid for the model. This effect is reached as a result of the ABS valve throttling.

\subsection{Pneumatic brake system equations}

The specific configuration of this system considers brake disks, which hold the wheels, as a result of the increment of the air pressure in the brake cylinder (Fig. 1). The entrance of the air trough the pipes from the central reservoir and the expulsion from the brake cylinder to the atmosphere is regulated by a common valve. This valve allows only one pipe to be open, when 1 is open 2 is closed and vice versa. The time response of the valve is considered small, compared with the time constant of the pneumatic system. Lets consider Fig. 1, we suppose the brake torque $T_{b}$ is proportional to the pressure $P_{b}$ in the brake cylinder

$$
T_{b}=k_{b} P_{b}
$$


with $k_{b}>0$. For the brake system we use an approximated model of pressure changes in the brake cylinder due to the opening of the valve with a first order relation (see Clover and Bernard [1998]), this relation can be represented as

$$
\tau \dot{P}_{b}+P_{b}=P_{c} u
$$

where $\tau$ is the time constant of the pipelines, $P_{c}$ is the pressure inside the central reservoir, $u$ is the valve input signal. We suppose that opening and closing of the valve is momentary and the parameters of the equation (2) are given by the following rules:

- When pipe 1 is opened and 2 is closed then $u=1$ and $\tau=\tau_{\text {in }}$

- When pipe 2 is opened and 1 is closed then $u=0$ and $\tau=\tau_{\text {out }}$

when pipe 2 is open the pressure into the brake cylinder is the atmospheric pressure $P_{a}$ which is considered equal to zero.



Fig. 1. Pneumatic brake system

\subsection{Wheel motion equations}

To describe the wheels motion we will use a partial mathematical model of the dynamic system as is done in Novozhilov et al. [2000], Kruchinin et al. [2001], Petersen et al. [2001] and Magomedov et al. [2001]. Consider Fig. 2 , the dynamics of the angular momentum change relative to the rotation axis are given by

$$
J \dot{\omega}=r f-B_{b} \omega-T_{b}
$$

where $\omega$ is the wheel angular velocity, $J$ is the wheel inertia moment, $r$ is the wheel radius, $B_{b}$ is a viscous friction coefficient and $f$ is the contact force of the wheel.

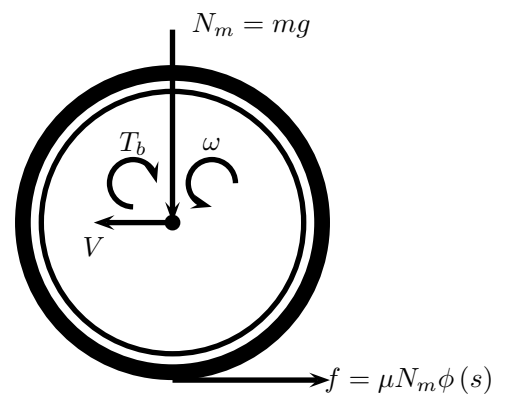

Fig. 2. Wheel forces and torques
The expression for longitudinal component of the contact force in the motion plane is

$$
f=\nu N_{m} \phi(s)
$$

where $\nu$ is the nominal friction coefficient between the wheel and the road, $N_{m}$ is the normal reaction force in the wheel

$$
N_{m}=m g
$$

with $m$ equal to the mass supported by the wheel and $g$ is the gravity acceleration. The function $\phi(s)$ represents a friction/slip characteristic relation between the tyre and road surface. Here, we use the Pacejka model given in Bakker et al. [1989], defined as follows

$$
\begin{aligned}
& \phi(s)= \\
& D \sin (C \arctan (B s-E(B s-\arctan (B s))))
\end{aligned}
$$

in general, this model produces a good approximation of the tyre/road friction interface. With the following parameters $B=10, C=1.9, D=1$ and $E=0.97$ that function represents the friction relation under a dry surface condition. A plot of this function is shown in Fig. 3



Fig. 3. Characteristic function $\phi(s)$

The slip rate $s$ is defined as

$$
s=\frac{v-r \omega}{v}
$$

where $v$ is the longitudinal velocity of the wheel mass center. The equations (3)-(5) characterize the wheel motion.

\subsection{The vehicle motion equation}

The vehicle longitudinal dynamics without lateral motion considered are represented as

$$
M \dot{v}=-F-F_{a}
$$

where $M$ is the vehicle mass; $F_{a}$ is the aerodynamic drag force, which is proportional to the vehicle velocity and is defined as

$$
F_{a}=\frac{1}{2} \rho C_{d} A_{f}\left(v+v_{w}\right)^{2}
$$

where $\rho$ is the air density, $C_{d}$ is the aerodynamic coefficient, $A_{f}$ is the frontal area of vehicle, $v_{w}$ is the wind velocity; the contact force of the vehicle $F$ is modeled of the form

$$
F=\mu N_{M}
$$

where $N_{M}$ is the normal reaction force of the vehicle, $N_{M}=M g$ with $M$ equal to the vehicle mass and $\mu=$ $\nu \phi(s)$. 
The dynamic equations of the whole system (2)-(7) can be rewritten using the state variables $\mathbf{x}=\left[x_{1}, x_{2}, x_{3}\right]^{T}=$ $\left[\omega, P_{b}, v\right]^{T}$ with initial conditions $x_{0}=x(0)$ results the following form:

$$
\begin{aligned}
& \dot{x}_{1}=-a_{0} x_{1}+a_{1} f(s)-a_{2} x_{2} \\
& \dot{x}_{2}=-a_{3} x_{2}+b u \\
& \dot{x}_{3}=-a_{4} F(s)-f_{w}\left(x_{3}\right)
\end{aligned}
$$

with output

$$
y=x_{1}
$$

where $a_{0}=B / J, a_{1}=r / J, a_{2}=k_{b} / J, a_{3}=1 / \tau, a_{4}=$ $1 / M, b=P_{c} / \tau$ and $f_{w}\left(x_{3}\right)=\frac{1}{2 M}\left(\rho C_{d} A_{f}\right)\left(x_{3}+v_{w}\right)^{2}$.

\section{SLIDING MODE REGULATOR FOR ABS}

Given $s^{*}(t)$ as the desired trajectory of the relative slip $s$, which must be close to maximize the function $\phi(s)$, the considered problem is to design a controller that obtains reference tracking in despite of the perturbations in the system. As a solution, we propose a Sliding Mode Block controller based in Loukianov [1998] for system (8). In addition, we introduce an estimation term for the steady state vehicle velocity in order to stabilize these dynamics.

Throughout the development of the controller, we will assume that all the state variables are available for measurement.

\subsection{Control Design}

Let $z$ be an estimate of the steady state of vehicle velocity residual dynamics $x_{3}$ and $s^{*}(t)$ be a twice differentiable function. Taking in account the direct action of the pressure $P_{b}$ in the brake cylinder over the wheels motion, we define the output tracking error as

$$
e_{1} \triangleq x_{1}-\frac{1-s^{*}}{r} x_{3}
$$

the estimation error

$$
e_{3} \triangleq x_{3}-z
$$

and the manifold

$$
s_{1} \triangleq e_{1}+k_{3} e_{3} .
$$

Hence, from (8) and (11) the derivative of $s_{1}$ is

$$
\dot{s}_{1}=f_{1}\left(x_{1}, x_{3}\right)+b_{1}\left(x_{1}, x_{3}\right) x_{2}-k_{3} \dot{z}+\Delta_{1}
$$

where $f_{1}\left(x_{1}, x_{3}\right)=\frac{k_{3} r+1-s^{*}}{r}\left[a_{4} F(s)-f_{w}\left(x_{3}\right)\right]-a_{0} x_{1}+$ $a_{1} f(s)$ and $b_{1}\left(x_{1}, x_{3}\right)=-a_{2}$. The term $\Delta_{1}$ contains the reference derivative $\dot{s}^{*}$, the variations of the friction parameter $\nu$, the wind speed $v_{w}$ and will be considered as an unmatched and bounded perturbation term.

Considering the variable $x_{2}$ as virtual control in (12) we determinate its desired value $x_{2 \delta}$ as

$$
x_{2 \delta}=x_{2 \delta, 0}+x_{2 \delta, 1}
$$

where $x_{2 \delta, 0}$ is the nominal part of the nominal control and $x_{2 \delta, 1}$ will be designed using the SM technique to attenuate the perturbation $\Delta_{1}$ in (12)

In this way, we propose the desired dynamics $-k_{0} s_{0}-k_{1} s_{1}$, which are introduced by means of

$$
x_{2 \delta, 0}=-\frac{1}{b_{1}\left(x_{1}, x_{3}\right)}\left[f_{1}\left(x_{1}, x_{3}\right)+k_{0} s_{0}+k_{1} s_{1}+k_{3} \dot{z}\right]
$$

where $k_{0}>0, k_{1}>0$ and $s_{0}$ is defined by

$$
\dot{s}_{0}=s_{1}, s_{0}(0)=0
$$

Now, in order to attenuate the perturbation term $\Delta_{1}$ in (12), we define the surface

$$
\sigma_{1}=s_{1}+\zeta
$$

where $\zeta$ is an SM integral variable and will be defined later. From (12), (14), (13) and (16) the derivative of $\sigma_{1}$ is given by

$$
\dot{\sigma}_{1}=-k_{0} s_{0}-k_{1} s_{1}+x_{2 \delta, 1}+\Delta_{1}+\dot{\zeta}
$$

Selecting $\dot{\zeta}=k_{0} s_{0}+k_{1} s_{1}$ with $\zeta(0)=-s_{1}(0)$, the eq. (17) reduces to

$$
\dot{\sigma}_{1}=x_{2 \delta, 1}+\Delta_{1}
$$

To enforce quasi-sliding motion in (18) the term $x_{2 \delta, 1}$ in (17) is chosen as

$$
x_{2 \delta, 1}=-k_{\sigma_{1}} \operatorname{sigm}\left(\varepsilon, \sigma_{1}\right)
$$

where we use the result that the sign function can be approximated by the sigmoid function in the form

$$
\lim _{\varepsilon \rightarrow \infty} \operatorname{sigm}(\varepsilon ; x)=\operatorname{sign}(x)
$$

Now we define a new error variable $e_{2}$ in the form

$$
e_{2}=x_{2 \delta}-x_{2}
$$

Using (8) and (19), straightforward calculations reveal

$$
\dot{e}_{2}=\Delta_{2}+b u
$$

where the term

$$
\Delta_{2}=a_{3} x_{2}+\frac{\partial x_{2 \delta}}{\partial x_{1}} \dot{x}_{1}+\frac{\partial x_{2 \delta}}{\partial x_{3}} \dot{x}_{3}
$$

is considered as a matched perturbation.

Defining $s_{2} \triangleq e_{2}$, to induce sliding mode on the manifold $s_{2}=0$ we choose the control signal as

$$
u=-\frac{1}{2} \operatorname{sign}\left(s_{2}\right)-\frac{1}{2}
$$

\subsection{Stability analysis}

Using the new variables $s_{0}, s_{1}, s_{2}$ and $\sigma_{1}$ the extended closed loop system (12), (15), (18) and (20) is presented as

$$
\begin{aligned}
& \dot{s}_{0}=s_{1} \\
& \dot{s}_{1}=-k_{0} s_{0}-k_{1} s_{1}+s_{2}-k_{\sigma_{1}} \operatorname{sigm}\left(\varepsilon, \sigma_{1}\right)+\Delta_{1} \\
& \dot{\sigma}_{1}=-k_{\sigma_{1}} \operatorname{sigm}\left(\varepsilon, \sigma_{1}\right)+\Delta_{1} \\
& \dot{s}_{2}=\Delta_{2}+b u \\
& \dot{x}_{3}=-a_{4} F-f_{w}\left(x_{3}\right)
\end{aligned}
$$

The stability of (22) - (26) closed loop by (21) is outlined in a step by step procedure:

Step A) Reaching phase of the projection motion (25);

Step B) SM stability of the projection motion (24)

Step C) SM Stability of (22)-(23);

Step D) Stability of residual dynamics (26).

We use the following assumptions:

$$
\begin{aligned}
& \left|\Delta_{1}\right| \leq \alpha_{1}\left|s_{1}\right|+\beta_{1} \\
& \left|\dot{\Delta}_{1}\right| \leq \alpha_{0}\left|\dot{\sigma}_{1}\right| \\
& \left|\Delta_{2}\right| \leq \beta_{2}
\end{aligned}
$$


and

$\alpha_{0}>0, \alpha_{1}>0, \beta_{1}>0, \beta_{2}>0, a_{3}>\alpha_{2}, b>\beta_{2}$.

With $i=1,2$, the terms $\alpha_{i}\left|s_{i}\right|$ and $\alpha_{0}\left|\dot{\sigma}_{1}\right|$ are used to represent the parametric variations and uncentain, and the terms $\beta_{i}$ represent external disturbances.

Step A) For the system (25) we use the Lyapunov function candidate $V_{2}=\frac{1}{2} e_{2}^{2}$ to analyze the stability conditions. The derivative of $V_{2}$ with respect to time is

$$
\dot{V}_{2}=e_{2}\left(\Delta_{2}-b\left(\frac{1}{2} \operatorname{sign}\left(s_{2}\right)-\frac{1}{2}\right)\right)
$$

under condition (29) we have

$$
\dot{V}_{2} \leq-\left|e_{2}\right|\left(b-\beta_{2}\right)
$$

Using (30), the system (25) is finite time stable.

Step B) To analyze the stability of the projection motion (24) we assume that the signum function can be approximated by the sigmoid function in the form $\operatorname{sigm}\left(\varepsilon_{1} ; x\right) \rightarrow \operatorname{sign}(x)$ as $\varepsilon_{1} \rightarrow \infty$, then, we can establish the following equality

$$
\operatorname{sign}(x)-\operatorname{sigm}\left(\varepsilon_{1} ; x\right)=\Delta_{s}\left(\varepsilon_{1} ; x\right)
$$

It is evidently that $\Delta_{s}(x)$ is bounded, that is, for a given $\varepsilon_{1}$ there is a positive constant $0<\gamma<1$ such that $\left\|\Delta_{s}\left(\varepsilon_{1} ; x\right)\right\|=\gamma$. Now, taking the Lyapunov candidate $V_{1}=\frac{1}{2} \sigma_{1}^{2}$ and taking its derivative, with (28) results

$$
\begin{aligned}
\dot{V}_{1} & =\sigma_{1}\left[-k_{\sigma_{1}} \operatorname{sigm}\left(\varepsilon_{1} ; \sigma_{1}\right)+\Delta_{1}\right] \\
& \leq-\left|\sigma_{1}\right|\left[k_{\sigma_{1}}(1-\gamma)-\alpha_{1}\left|\sigma_{1}\right|-\beta_{1}\right]
\end{aligned}
$$

therefore, if $k_{\sigma_{1}}>\frac{\beta_{1}}{1-\gamma}$ then $\sigma_{1}$ converges to a vicinity of zero, $\left|\sigma_{1}\right|<\vartheta_{1}$, with

$$
\vartheta_{1}=\frac{\ln \left(\frac{2-\gamma}{\gamma}\right)}{2 \varepsilon_{1}}
$$

and, with (28), $\dot{\sigma}_{1}$ converges to zero in finite time González-Jiménez and Loukianov [2008].

Step C) To analyze the SM motion on the manifold $s_{2}=0$, and in the vecinity of $\sigma_{1}=0$ described by (22)$(23)$, that system can be regarded as a linear system with nonvanishing perturbation in the form:

$$
\dot{\eta}=\mathbf{A} \eta+\mathbf{D}(\eta)
$$

where

$$
\eta=\left[\begin{array}{ll}
s_{0} & s_{1}
\end{array}\right]^{T} ; \mathbf{A}=\left[\begin{array}{cc}
0 & 1 \\
-k_{0} & -k_{1}
\end{array}\right] ; \mathbf{D}(\eta)=\left[\begin{array}{c}
0 \\
\Delta_{1}
\end{array}\right]
$$

and the perturbation term is bounded by $\|\mathbf{D}(\eta)\| \leq$ $\alpha_{1}\left|s_{1}\right|+\beta_{1}$. Now we use the following Lyapunov candidate function:

$$
V_{\eta}=\frac{1}{2} \eta^{T} \mathbf{P} \eta
$$

with $\mathbf{P}$ positive definite. With the correct selection of the elements $k_{0}$ and $k_{1}$ the matrix $A$ is Hurwitz, then exists one unique solution $(\mathbf{P}>0)$ to the Lyapunov equation

$$
\mathbf{A}^{T} \mathbf{P}+\mathbf{P A}=-\mathbf{Q}
$$

where $\mathbf{Q}=\mathbf{Q}^{T}, \mathbf{Q}>0$. Derivating (33) we obtain

$$
\dot{V}_{\eta}=-\eta^{T} \mathbf{Q} \eta-2 \eta^{T} \mathbf{P D}(\eta)
$$

which is bounded by

$$
\begin{aligned}
\dot{V}_{\eta} & =-\eta^{T} \mathbf{Q} \eta-2 \eta^{T} \mathbf{P D}(\eta) \\
& \leq-\lambda_{\min }(\mathbf{Q})\|\eta\|_{2}^{2}+2 \lambda_{\max }(\mathbf{P})\|\eta\|_{2}\left(\alpha_{1}\|\eta\|_{2}+\beta_{1}\right) \\
& \leq\left(-\lambda_{\min }(\mathbf{Q})+2 \alpha_{1} \lambda_{\max }(\mathbf{P})\right)\|\eta\|_{2}^{2}+2 \beta_{1} \lambda_{\max }(\mathbf{P})\|\eta\|_{2} \\
& =-\alpha(1-\theta)\|\eta\|_{2}^{2}-\alpha \theta\|\eta\|_{2}^{2}+\beta\|\eta\|_{2}
\end{aligned}
$$

where $\alpha=\lambda_{\min }(\mathbf{Q})-2 \alpha_{1} \lambda_{\max }(\mathbf{P})$ and $\beta=2 \beta_{1} \lambda_{\max }(\mathbf{P})$, then

$$
\dot{V}_{1} \leq-\alpha(1-\theta)\|\eta\|_{2}^{2}
$$

for $\forall \eta:\|\eta\|_{2}>\frac{\beta}{\alpha \theta}=\delta_{\eta}$.

Thus, the solution $\eta(t)$ of (32) is ultimately bounded and the ultimate bound is given by

$$
\|\eta\|_{2} \leq \delta_{\eta} \frac{\sqrt{\lambda_{\max }(\mathbf{P})}}{\sqrt{\lambda_{\min }(\mathbf{P})}}
$$

Step D) For the residual dynamics $x_{3}$ we have

$$
\dot{x}_{3}=-a_{4} F(s)-f_{w}\left(x_{3}\right)
$$

then, defining

$$
\begin{aligned}
& g=-a_{4} F(s)-f_{w}\left(x_{3}\right) \\
& a=\left.\frac{\partial g}{\partial x_{3}}\right|_{x_{3}=0, s=s^{*}} \\
& \lambda=\left.\frac{\partial g}{\partial s}\right|_{x_{3}=0, s=s^{*}}
\end{aligned}
$$

yields

$$
\dot{x}_{3}=a x_{3}+\lambda s+\varphi
$$

where $\varphi$ contains the nonlinear terms. Hence, the dynamics for $e_{3}$ result in

$$
\dot{e}_{3}=a e_{3}+a z+\lambda s+\varphi-\dot{z}
$$

Finally, defining the sigmoidal function $\operatorname{sigm}\left(\varepsilon_{2} ; x\right) \triangleq$ $\tanh \left(\varepsilon_{2} x\right)$, and taking

$$
\dot{z}=a z+\lambda s-e_{1}+k_{4} \operatorname{sigm}\left(\varepsilon_{2} ; e_{3}\right)
$$

results

$$
\dot{e}_{3}=a e_{3}+e_{1}-k_{4} \operatorname{sigm}\left(\varepsilon_{2} ; e_{3}\right)+\Delta_{3}
$$

where $\Delta_{3} \triangleq \varphi$ will be considered as a nonvanishing perturbation term

$$
\left|\Delta_{3}\right| \leq \alpha_{3}\left|e_{3}\right|+\beta_{3}
$$

In sliding motion we have $s_{1}=0$, that gives

$$
\dot{e}_{3}=\left(a-k_{3}\right) e_{3}-k_{4} \operatorname{sigm}\left(e_{3} ; \varepsilon_{2}\right)+\Delta_{3}
$$

Now, assuming that the sign function can be approximated by the sigmoid function in the form

$$
\lim _{\varepsilon_{2} \rightarrow \infty} \operatorname{sigm}\left(\varepsilon_{2} ; x\right)=\operatorname{sign}(x)
$$

we can establish the following equality

$$
\operatorname{sign}(x)-\operatorname{sigm}\left(\varepsilon_{2} ; x\right)=\Delta_{s}\left(\varepsilon_{2} ; x\right)
$$

It is evidently that $\Delta_{s}(x)$ is bounded, that is, for a given $\varepsilon_{2}$ there is a positive constant $0<\delta<1$ such that

$$
\left\|\Delta_{s}\left(\varepsilon_{2} ; x\right)\right\|=\delta
$$

Taking the Lyapunov candidate

$$
V_{3}=\frac{1}{2} e_{3}^{2}
$$

and taking its derivative

$$
\begin{aligned}
\dot{V}_{3} & =e_{3}\left[\left(a-k_{3}\right) e_{3}-k_{4} \operatorname{sigm}\left(\varepsilon_{2} ; e_{3}\right)+\Delta_{3}\right] \\
& \leq-e_{3}^{2}\left(k_{3}-a-\alpha_{3}\right)-\left|e_{3}\right|\left[k_{4}(1-\delta)-\beta_{3}\right]
\end{aligned}
$$


therefore, if $k_{3}>a+\alpha_{3}$ and $k_{4}>\beta_{3} /(1-\delta)$ then $e_{3}$ converges to a vicinity of zero, $\left|e_{3}\right|<\vartheta_{2}$, with

$$
\vartheta_{2}=\frac{\ln \left(\frac{2-\delta}{\delta}\right)}{2 \varepsilon_{2}}
$$

and from (11), $e_{1}$ converges to a vicinity of zero, $\left|e_{1}\right|<$ $\vartheta_{2} / k_{3}$.

\section{SIMULATION RESULTS}

To show the effectiveness of the proposed control law, simulations have been carried out on one wheel model design example, the system parameters used are listed in Table 1.

\begin{tabular}{|c|c|c|c|}
\hline \multicolumn{5}{|c|}{ TABLE 1 } \\
\hline Values of Parameters (MKS Units) \\
\hline Parameter & Value & Parameter & Value \\
\hline$A_{f}$ & 6.6 & $V_{w}$ & -6 \\
\hline$P_{c}$ & 8 & $v$ & 0.5 \\
\hline$M$ & 1800 & $B$ & 10 \\
\hline$J$ & 18.9 & $C$ & 1.9 \\
\hline$R$ & 0.535 & $D$ & 1 \\
\hline$m$ & 450 & $E$ & 0.97 \\
\hline$\rho$ & 1.225 & $g$ & 9.81 \\
\hline$C_{d}$ & 0.65 & $B_{b}$ & 0.08 \\
\hline
\end{tabular}

In order to maximize the friction force, we suppose that slip tracks a constant signal during the simulations.

$$
y_{\text {ref }}=0.203
$$

which produces a value close to the maximum of the function $\phi(s)$. The parameters used in the control law are $k_{0}=700, k_{1}=120, k_{3}=2, k_{4}=100$ and $\varepsilon_{1}=\varepsilon_{2}=100$.

On the other hand, to show robustness property of the control algorithm in presence of parametric variations we introduce a change of the friction coeficient $\nu$ which produces different contact forces, namely $F$ and $\hat{F}$. Then, $\nu=0.5$ for $t<1 \mathrm{~s}, \nu=0.52$ for $t \in[1,2.5) \mathrm{s}$, and $\nu=0.5$ for $t \geq 2.5 \mathrm{~s}$. It is worth mentioning that just the nominal values were considered in the control design.

In Fig. 4 the slip performance trough the simulation is shown, Fig. 5 shows the friction function behavior $\phi(s)$ during the braking process



Fig. 4. Slip performance in the braking process



Fig. 5. Performance of $\phi(s)$ in the braking process

while Fig. 6 and Fig. 7 summarize the behavior of the error variable $e_{1}$ and $e_{2}$, respectively.



Fig. 6. Tracking error $e_{1}=s-s^{*}$

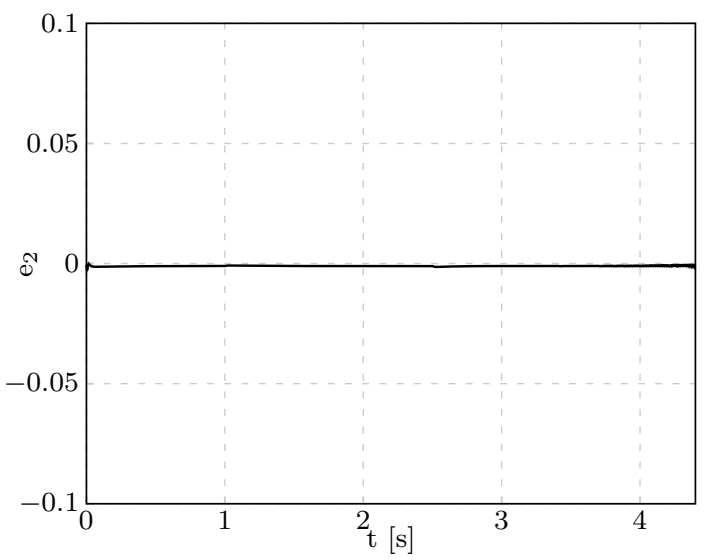

Fig. 7. Error on the sliding surface $e_{2}=x_{2 \delta}-x_{2}$ 


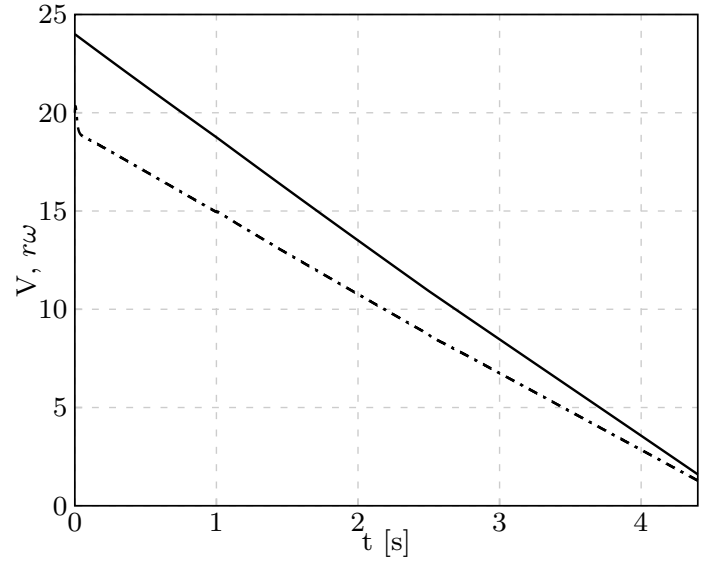

Fig. 8. Longitudinal speed $V$ (solid) and linear wheel speed $r \omega($ dashed $)$

In Fig. 8 the longitudinal speed $v$ and the linear wheel speed $r \omega$ are showed; it is worth noting that the slip controller should be turn off when the longitudinal speed $v$ is close to zero. In Fig. 9 the control action is shown.

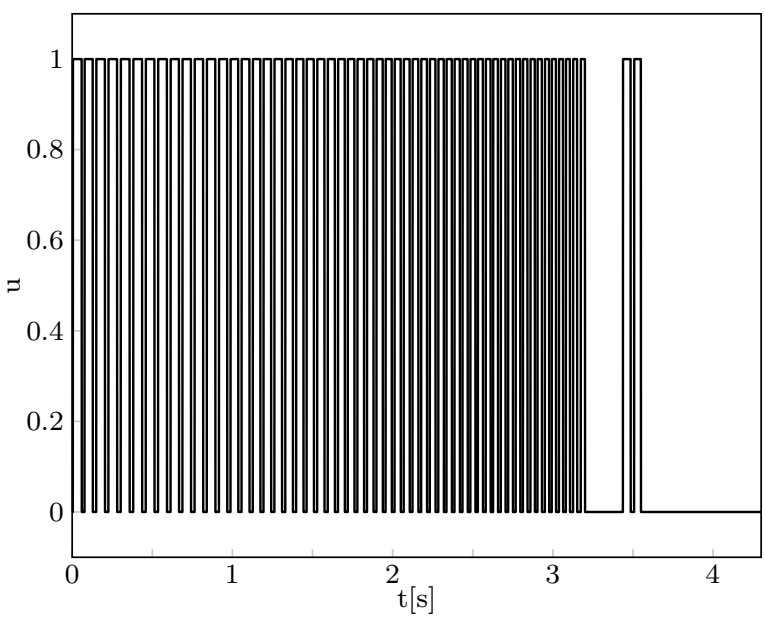

Fig. 9. Control input $u$

Finally, in Fig. 10 the nominal $F$, and the $\hat{F}$ contact force are shown.



Fig. 10. Nominal $F$ (solid) and $\hat{F}$ (dashed) vehicle contact forces

\section{CONCLUSION}

In this work a sliding mode regulator based in block control for ABS has been proposed. The simulation results show good performance and robustness of the closed-loop system in presence of both the matched and unmatched perturbations, namely, parametric variations and neglected dynamics.

\section{REFERENCES}

E. Bakker, H. Pacejka, and L. Lidner. A new tire model with application in vehicle dynamic studies. SAE Paper No. 890087, 01:101-113, 1989.

C.L. Clover and J.E. Bernard. Longitudinal tire dynamics. Vehicle System Dynamics, 29:231-259, 1998.

S.V. Drakunov, U. Ozguner, P. Dix, and B. Ashrafi. ABS control using optimum search via sliding modes. IEEE Transactions on Control Systems Technology, 3(1):7985, 1995.

L.E González-Jiménez and A.G. Loukianov. Integral nested sliding mode control for robotic manipulators. In Proc. of the 17th IFAC World Congress pp. 9899-9904, Seoul, Korea, July 6-11, 2008.

A.El. Hadri, J.C. Cadiou, and N.K. MSirdi. Adaptive sliding mode control for vehicle traction. In Proc. of the 15th IFAC World Congress, Barcelona, Spain, July 22262002.

P.A. Kruchinin, M. Magomedov, and I.V. Novozhilov. Mathematical model of an automobile wheel for antilock modes of motion. Mechanics of Solids, 36(6):52-57, 2001.

A.G. Loukianov. Nonlinear block control with sliding mode. Automation and Remote Control, 59(7):916-933, 1998.

M. Magomedov, V.V. Alexandrov, and K.A. Pupkov. Robust adaptive stabilization of moving a car under braking with ABS in control circuit. In SAE Store. ATTCE, editor, Automotive and Transportation Technology Congress and Exposition Proceedings - Chassis and Total Vehicle, Barcelona, Spain., volume 6, 2001.

Wu Ming-Chin and Shih Ming-Chang. Simulated and experimental study of hydraulic anti-lock braking system using sliding-mode PWM control. Mechatronics, 13:331-351, 2003.

I.V. Novozhilov, P.A. Kruchinin, and M. Magomedov. Contact force relation between the wheel and the contact surface. Collection of scientific and methodic papers Teoreticheskaya mekhanika, MSU, 23:86-95, 2000. (In Russian).

I.T. Petersen, A. Johansen, J. Kalkkuhl, and J. Ludemann. Wheel slip control in ABS brakes using gain scheduled constrianed LQR. In Proc. European Contr. Conf., Porto., 2001.

H.S. Tan and Y.K. Chin. Vehicle traction control: variable structure control approach. Journal of Dynamic Systems, measurement and Control, 113:223-230, 1991.

C. Unsal and K. Pushkin. Sliding mode measurement feedback control for antilock braking systems. IEEE Transactions on Control Systems Technology, 7(2):271$278,1999$. 